\title{
On Twin Edge Colorings of Finite 2-Dimensional Grids
}

\section{Huan Yanga, Shuang Liang Tian, ${ }^{\mathrm{b},}$, Xia Hong Caic and Su Su Jiaod}

Northwest Minzu University, College of Mathematics and Computer Science, Lanzhou, Gansu, China

ayanghuan082@126.com, bsl_tian@163.com, c1850697869@qq.com, d1226062170@qq.com

${ }^{*}$ Corresponding author

Keywords: Twin edge coloring, Twin chromatic number, Finite 2-dimensional grids.

Abstract. Let $\sigma$ be a proper edge coloring of a connected graph $G$ of order at least 3, where the color set is $\{0,1,2, \cdots, k-1\}$. If $\sigma$ can induce a proper vertex coloring of $G$, then $\sigma$ is called a twin edge $k$-coloring of $G$. The minimum number of colors for which $G$ has a twin edge coloring is called the twin chromatic index of $G$. In this paper, twin edge colorings of finite 2-dimensional grids are studied, and it's twin chromatic number is obtained.

\section{有限2-维网格的孪生边染色}

\section{杨环 ${ }^{a}$, 田双亮 ${ }^{\text {, }}{ }^{*}$, 蔡侠红 ${ }^{c}$, 焦素素 ${ }^{d}$}

西北民族大学数学与计算机科学学院, 兰州, 甘肃, 中国

ayanghuan082@126.com, bsl_tian@163.com, c1850697869@qq.com, d1226062170@qq.com

\section{通讯作者}

关键词: 有限2-维网格; 孪生边染色; 孪生边染色数

中文摘要. 设 $\sigma$ 是一个阶至少为 3 的连通图 $G$ 的 $k$-正常边染色, 其中颜色集合为 $\{0,1,2, \cdots, k-1\}$. 若 $\sigma$ 能够诱导一个 $G$ 的正常点染色, 则称 $\sigma$ 是 $G$ 的孪生 $k$-边染色. 最少的 $k$ 值为 $G$ 的孪生边色 数, 记为 $\chi_{t}^{\prime}(G)$. 本文研究了有限 $2-$ 维网格 $G(n, m)$ 的孪生边染色, 得到了相应的染色数.

\section{1 引言}

设 $G$ 为简单连通图, 分别用 $V(G)$ 和 $E(G)$ 表示 $G$ 的顶点集和边集, 并用 $\Delta(G)$ 表示 $G$ 的最大 度, $d(v)$ 和 $E_{v}$ 分别表示顶点 $v$ 的度数和 $v$ 的所有关联边构成的集合.

Andrews在文献 [1] 中提出了孪生边染色的概念，并得到关于路、圈、完全图以及完全二 部图的孪生边色数. 所谓简单图 $G$ 的孪生 $k$-边染色 $\sigma$ 是指其诱导的点染色 $\sigma^{\prime}$ 是 $G$ 的正常点染 色的正常 $k$ - 边染色，其中 $\sigma$ 的颜色集合为 $\{0,1,2, \cdots, k-1\}$ ，且对任 意 $u \in V(G)$, $\sigma^{\prime}(u)=\left(\sum_{u v \in E_{v}} \sigma(u v)\right) \bmod k$, 最少的 $k$ 值为 $G$ 的孪生边色数, 记为 $\chi_{t}^{\prime}(G)$.

由孪生边染色概念, 显然以下引理成立.

引理 1.1 若 $G$ 是阶至少为3且存在相邻最大度点的简单连通图, 则 $\chi_{t}^{\prime}(G) \geq \Delta(G)+1$.

与孪生边染色密切相关的染色概念是邻点可区别边染色 ${ }^{[2]}$, 其中图 $G$ 的邻点可区别边染 色是指 $G$ 的任意相邻顶点具有不同色集的正常边染色，所用颜色数最少的值称为邻点可区别 边色数, 记为 $\chi_{a}^{\prime}(G)$. 
由邻点可区别边染色与孪生边染色概念可知, 图 $G$ 的任一 $k$-孪生边染色一定是 $G$ 的一个 $k$-邻点可区别边染色, 但反之不一定成立, 如3-阶路的2-邻点可区别边染色不是其孪生2-边 染色. 因此, 对阶至少为 3 的简单连通图 $G$, 有 $\chi_{a}^{\prime}(G) \leq \chi_{t}^{\prime}(G)$.

Baril等在文献 [2] 中研究了多维网格图和超立方体的邻点可区别边染色, 结果表明：多 维网格图和超立方体的邻点可区别边色数均等于它们的最大度加1. 另外, Zhang在文献 [3] 中 提出了邻点可区别边色数猜想 “设 $G$ 是阶至少为 3 的简单图, 且 $G \neq C_{5}$, 则 $\chi_{a}^{\prime}(G) \leq \Delta(G)+2$. ”, 并验证了路、圈、树、完全图、完全二部图等特殊图类满足此猜想. Balister和Gyori等人在文 献 [4] 中验证了对所有的二部图和最大度为 3 的图都满足此猜想. Dai和Bu在文献 [5]中给出了 邻点可区别边染色的一个上界：任意简单连通图 $G$ 的邻点可区别边色数不超过 $3 \Delta(G)-1$. Zhang 和Lin在文献 $[6]$ 中将此上界改进为 $\frac{5}{2}(\Delta(G)+2)$. Bu等在文献 $[7]$ 中证明了围长至少为 6 的 无孤立边的平面图的邻点可区别边色数不超过 $\Delta(G)+2$. 严丞超等在文献 [8] 中证明了围长至 少为 4 且最大度至少为 6 的平面图 $G$ 的邻点可区别边色数不超过 $\Delta(G)+2$. 更多的相关结果见 文献 $[9-14]$.

本文主要研究有限2-维网格 $G(n, m)$ 的孪生边染色, 其中 $G(n, m)$ 是指具有顶点集 $\{0,1, \cdots, n-1\} \times\{0,1, \cdots, m-1\}$ 的图, 且顶点 $(x, y)$ 与 $\left(x^{\prime}, y^{\prime}\right)$ 相邻当且仅当 $x=x^{\prime}$ 且 $\left|y-y^{\prime}\right|=1$, 或 $y=y^{\prime}$ 且 $\left|x-x^{\prime}\right|=1$. 文中未说明的符号和术语参见 [15、16].

\section{2 主要结果及证明}

设 $n, m \geq 2$, 关于 $G(n, m)$ 的孪生边色数, 有以下结果:

定理2. 1 若 $m=n=2, m=n=3$, 或 $m=2$ 且 $n \geq 3$, 则 $\chi_{t}^{\prime}(G(n, m))=4$.

证明 若 $m=n=2$, 则 $G(n, m)$ 为 4 -阶圈, 显然, $\chi_{t}^{\prime}(G(n, m))=4$. 若 $m=n=3$, 或 $m=2$ 且 $n \geq 3$, 则分以下两种情况进行讨论.

情况 $1 \quad m=n=3$. 显然, $\chi_{t}^{\prime}(G(3,3)) \geq 4$, 所以仅需构造 $G(3,3)$ 的一个 $4-$ 孪生边染色.

设颜色集合为 $\{0,1,2,3\}$. 并令 $u_{1}=(0,0), u_{2}=(1,0), u_{3}=(2,0), u_{4}=(0,1), u_{5}=(1,1), u_{6}=(2,1)$, $u_{7}=(0,2), u_{8}=(1,2), u_{9}=(2,2)$. 构造 $G(3,3)$ 的染色 $\sigma$, 显然, $\sigma^{\prime}\left(u_{1}\right)=1, \sigma^{\prime}\left(u_{2}\right)=0, \sigma^{\prime}\left(u_{3}\right)=1$, $\sigma^{\prime}\left(u_{4}\right)=0, \sigma^{\prime}\left(u_{5}\right)=2, \sigma^{\prime}\left(u_{6}\right)=3, \sigma^{\prime}\left(u_{7}\right)=1, \quad \sigma^{\prime}\left(u_{8}\right)=3, \sigma^{\prime}\left(u_{9}\right)=1$. 容易验证, $\sigma^{\prime}$ 是 $G(3,3)$ 的一 个 $4-$ 孪生边染色. 因此, $\chi_{t}^{\prime}(G(3,3))=4$.

情况 $2 m=2$ 且 $n \geq 3$. 由引理 $1.1, \chi_{t}^{\prime}(G(n, 2)) \geq 4$. 为证明 $\chi_{t}^{\prime}(G(n, 2)) \leq 4$, 现在构造 $G(n, 2)$ 的一 个4-孪生边染色.

设颜色集合为 $\{0,1,2,3\}$, 并对 $x=0,1, \cdots, n-1, y=0,1$, 令

$$
\sigma((x, 0)(x, 1))=0, \quad \sigma((x, y)(x+1, y))=1+(x+y+1)_{3} .
$$

显然, $\sigma$ 所用的颜色数是 4 .

首先, 证明 $\sigma$ 是 $G(n, 2)$ 的正常边染色. 对 $G(n, 2)$ 的顶点 $(x, 0), x=0,1, \cdots, n-1$, 由 $\sigma$ 的定义, 可知该顶点的可能关联边的颜色为

$$
\sigma((x-1,0)(x, 0))=1+(x)_{3}, \quad \sigma((x, 0)(x+1,0))=1+(x+1)_{3}, \quad \sigma((x, 0)(x, 1))=0 .
$$

因 $\sigma((x-1,0)(x, 0)) \in\{1,2,3\}, \sigma((x, 0)(x+1,0)) \in\{1,2,3\}$, 所以， $\sigma((x-1,0)(x, 0)) \neq \sigma((x, 0)(x, 1))$, $\sigma((x, 0)(x+1,0)) \neq \sigma((x, 0)(x, 1))$. 若 $\sigma((x-1,0)(x, 0))=\sigma((x, 0)(x+1,0))$, 则 $1+(x)_{3}=1+(x+1)_{3}$, 即 $(0)_{3}=(1)_{3}$, 这是不可能的. 因此, $(x, 0)$ 的不同关联边具有不同的颜色. 类似地, 对任一点 $(x, 1)$, 其所有 可能关联边也具有不同的颜色. 由以上分析可知, $\sigma$ 是 $G(n, 2)$ 的正常边染色.

其次, 证明由 $\sigma$ 诱导的 $G(n, 2)$ 的点染色 $\sigma^{\prime}$ 是正常的. 任取 $G(n, 2)$ 的一个顶点 $(x, y)$, 分以下 两种情况讨论 $\sigma^{\prime}$ 是 $G(n, 2)$ 的正常点染色. 
假设 $y=0$. 由 $\sigma$ 及 $\sigma^{\prime}$ 的定义, 顶点 $(0,0)$ 及其邻点的颜色与 $(n-1,0)$ 及其邻点的颜色分别为

$$
\begin{aligned}
& \sigma^{\prime}(0,0)=2, \quad \sigma^{\prime}(1,0)=1, \quad \sigma^{\prime}(0,1)=3, \quad \sigma^{\prime}(n-1,0)=\left(1+(n-1)_{3}\right)_{4}, \\
& \sigma^{\prime}(n-2,0)=\left(2+(n-2)_{3}+(n-1)_{3}\right)_{4}, \quad \sigma^{\prime}(n-1,1)=\left(1+(n)_{3}\right)_{4} ;
\end{aligned}
$$

显然, $(0,0)$ 与其邻点具有不同的颜色. 假设 $\sigma^{\prime}(n-1,0)=\sigma^{\prime}(n-2,0)$, 则 $0=\left(1+(n+1)_{3}\right)_{4}$, 这是 不可能的, 因为 $1 \leq 1+(n+1)_{3} \leq 3$. 假设 $\sigma^{\prime}(n-1,0)=\sigma^{\prime}(n-1,1)$, 则可导出矛盾 $2=0$. 因此, $(n-1,0)$ 与 其邻点具有不同的颜色.

对顶点 $(x, 0), x=1,2, \cdots, n-2$, 由 $\sigma$ 及 $\sigma^{\prime}$ 的定义, $(x, 0)$ 及其邻点的颜色分别为

$$
\begin{aligned}
\sigma^{\prime}(x, 0) & =\left(2+(x)_{3}+(x+1)_{3}\right)_{4}, \quad \sigma^{\prime}(x-1,0)=\left(2+(x-1)_{3}+(x)_{3}\right)_{4}, \\
\sigma^{\prime}(x+1,0) & =\left(2+(x+1)_{3}+(x+2)_{3}\right)_{4}, \quad \sigma^{\prime}(x, 1)=\left(2+(x+1)_{3}+(x+2)_{3}\right)_{4} .
\end{aligned}
$$

假设 $\sigma^{\prime}(x, 0)=\sigma^{\prime}(x-1,0)$, 或 $\sigma^{\prime}(x, 0)=\sigma^{\prime}(x+1,0)$, 或 $\sigma^{\prime}(x, 0)=\sigma^{\prime}(x, 1)$, 则可导出矛盾 $1=2$ 或 $0=2$. 因此, $(x, 0)$ 与其邻点具有不同的颜色.

假设 $y=1$. 与 $y=0$ 的情形类似, 顶点 $(0,1)$ 及其邻点的颜色与 $(n-1,1)$ 及其邻点的颜色分别为

$$
\begin{gathered}
\sigma^{\prime}(0,1)=3, \quad \sigma^{\prime}(1,1)=0, \quad \sigma^{\prime}(0,0)=2, \quad \sigma^{\prime}(n-1,1)=\left(1+(n)_{3}\right)_{4}, \\
\sigma^{\prime}(n-2,1)=\left(2+(n-1)_{3}+(n)_{3}\right)_{4}, \quad \sigma^{\prime}(n-1,0)=\left(1+(n-1)_{3}\right)_{4} .
\end{gathered}
$$

显然, $(0,1)$ 与其邻点具有不同的颜色. 假设 $\sigma^{\prime}(n-1,1)=\sigma^{\prime}(n-2,1)$ 或 $\sigma^{\prime}(n-1,1)=\sigma^{\prime}(n-1,0)$,

则可导出矛盾 $0=\left(1+(n+2)_{3}\right)_{4}$ 或 $0=2$. 因此, $(n-1,1)$ 与其邻点具有不同的颜色.

对顶点 $(x, 1), x=1,2, \cdots, n-2$, 由 $\sigma$ 及 $\sigma^{\prime}$ 的定义, $(x, 1)$ 及其邻点的颜色分别为

$$
\begin{array}{cc}
\sigma^{\prime}(x, 1)=\left(2+(x+1)_{3}+(x+2)_{3}\right)_{4}, & \sigma^{\prime}(x-1,1)=\left(2+(x)_{3}+(x+1)_{3}\right)_{4}, \\
\sigma^{\prime}(x+1,1)=\left(2+(x+2)_{3}+(x)_{3}\right)_{4}, & \sigma^{\prime}(x, 0)=\left(2+(x)_{3}+(x+1)_{3}\right)_{4} .
\end{array}
$$

假设 $\sigma^{\prime}(x, 1)=\sigma^{\prime}(x-1,1)$, 或 $\sigma^{\prime}(x, 1)=\sigma^{\prime}(x, 0)$, 或 $\sigma^{\prime}(x, 1)=\sigma^{\prime}(x+1,1)$, 则可导出矛盾 $2=0$ 或 $1=2$. 因 此, $(x, 1)$ 与其邻点具有不同的颜色.

由以上分析, $\sigma^{\prime}$ 是 $G(n, 2)$ 的正常点染色.

定理2. 2 设任意整数 $n, m \geq 3$, 且满足 $(n)_{3}=2$ 或 $(m)_{3}=2$, 则 $\chi_{t}^{\prime}(G(n, m))=5$.

证明 因 $G(n, m) \cong G(m, n)$, 所以不妨假设 $(n)_{3}=2$. 由引理 $1.1, \chi_{t}^{\prime}(G(n, m)) \geq 5$, 所以仅需构 造 $G(n, m)$ 的一个 $5-$ 孪生边染色.

设颜色集合为 $\{0,1,2,3,4\}$, 并对 $x=0,1, \cdots, n-1, y=0,1, \cdots, m-1$, 令

$$
\sigma((x, y)(x, y+1))=(y+1)_{2}, \quad \sigma((x, y)(x+1, y))=2+\left(x-(y)_{2}\right)_{3} .
$$

显然, $\sigma$ 所用的颜色数是 5 .

首先, 证明 $\sigma$ 为 $G(n, m)$ 的正常边染色. 由 $\sigma$ 的定义可知, $G(n, m)$ 任一顶点 $(x, y)$ 的所有可能 关联边的颜色为

$$
\begin{gathered}
\sigma((x, y)(x+1, y))=2+\left(x-(y)_{2}\right)_{3}, \quad \sigma((x, y)(x, y+1))=(y+1)_{2}, \\
\sigma((x-1, y)(x, y))=2+\left(x-(y)_{2}-1\right)_{3}, \quad \sigma((x, y-1)(x, y))=(y)_{2} .
\end{gathered}
$$

显 然 , $\{\sigma((x, y)(x+1, y)), \sigma((x-1, y)(x, y))\} \cap\{\sigma((x, y)(x, y+1)), \sigma((x, y-1)(x, y))\}=\phi \quad$, 且 $\sigma((x, y)(x, y+1)) \neq \sigma((x, y-1)(x, y))$. 假设 $\sigma((x, y)(x+1, y))=\sigma((x-1, y)(x, y))$, 则导出矛盾 $0=2$. 因此, $(x, y)$ 的不同关联边具有不同的颜色, 即 $\sigma$ 是 $G(n, m)$ 的正常边染色.

其次, 证明由 $\sigma$ 诱导的 $G(n, m)$ 的点染色 $\sigma^{\prime}$ 是正常的. 任取 $G(n, m)$ 的两个相邻顶点 $u$ 与 $v$, 不妨设 $u=(x, y)$ 且 $d(u) \leq d(v)$, 其中 $x=0,1, \cdots, n-1, y=0,1, \cdots, m-1$. 根据 $G(n, m)$ 的结构可知, $v=(x \pm 1, y)$ 或 $v=(x, y \pm 1)$. 现在需要证明 $\sigma^{\prime}(u) \neq \sigma^{\prime}(v)$, 考虑 4 种情况: (i) $d(u)=d(v)=4$; (ii) $d(u)=2$ 且 $d(v)=3$; (iii) $d(u)=3$ 且 $d(v)=4$; (iv) $d(u)=d(v)=3$.

情况 $1 d(u)=d(v)=4$. 由 $\sigma$ 及 $\sigma^{\prime}$ 的定义, 有

$$
\sigma^{\prime}(u)=\sigma^{\prime}(x, y)=\left(x-(y)_{2}+2\right)_{3}+\left(x-(y)_{2}\right)_{3}
$$

当 $v=(x \pm 1, y)$ 时, 由公式 (1) 可知, 


$$
\sigma^{\prime}(v)=\sigma^{\prime}(x \pm 1, y)=\left(x \pm 1-(y)_{2}+1\right)_{3}+\left(x \pm 1-(y)_{2}\right)_{3} .
$$

假设 $\sigma^{\prime}(u)=\sigma^{\prime}(v)$, 则产生矛盾 $2=1$ 或 $0=1$. 因此, $\sigma^{\prime}(u) \neq \sigma^{\prime}(v)$.

类似地, 当 $v=(x, y \pm 1)$ 时, 由公式 (1) 可知,

$$
\sigma^{\prime}(v)=\sigma^{\prime}(x, y \pm 1)=\left(x-(y \pm 1)_{2}+2\right)_{3}+\left(x-(y \pm 1)_{2}\right)_{3} .
$$

假设 $\sigma^{\prime}(u)=\sigma^{\prime}(v)$, 则产生矛盾 $0=1$. 因此, $\sigma^{\prime}(u) \neq \sigma^{\prime}(v)$.

情况 $2 d(u)=2$ 且 $d(v)=3$. 则 $u=(0,0)$, 或 $u=(n-1,0)$, 或 $u=(0, m-1)$, 或 $u=(n-1, m-1)$.

当 $u=(0,0)$ 时, 由 $\sigma$ 及 $\sigma^{\prime}$ 的定义, 顶点 $u$ 及其相邻点在 $\sigma^{\prime}$ 下的颜色分别为

$\sigma^{\prime}(0,0)=3, \quad \sigma^{\prime}(1,0)=1, \quad \sigma^{\prime}(0,1)=0$.

显然, $u$ 与其相邻顶点具有不同颜色.

当 $u=(n-1,0)$ 时, 由 $\sigma$ 及 $\sigma^{\prime}$ 的定义, 顶点 $u$ 及其相邻点在 $\sigma^{\prime}$ 下的颜色分别为

$$
\sigma^{\prime}(n-1,0)=\left(3+(n+1)_{3}\right)_{5}, \quad \sigma^{\prime}(n-2,0)=\left((n)_{3}+(n+1)_{3}\right)_{5}, \quad \sigma^{\prime}(n-1,1)=\left(3+(n)_{3}\right)_{5} .
$$

假设 $\sigma^{\prime}(n-1,0)=\sigma^{\prime}(n-2,0)$, 则可导出矛盾 $3=(n)_{3}$; 假设 $\sigma^{\prime}(n-1,0)=\sigma^{\prime}(n-1,1)$, 则可导出矛盾 $1=0$.

当 $u=(0, m-1)$ 时, 由 $\sigma$ 及 $\sigma^{\prime}$ 的定义, 顶点 $u$ 及其相邻点在 $\sigma^{\prime}$ 下的颜色分别为

$$
\begin{gathered}
\sigma^{\prime}(0, m-1)=\left(2+(m+1)_{2}+\left(-(m+1)_{2}\right)_{3}\right)_{5}, \quad \sigma^{\prime}(0, m-2)=\left(3+\left(-(m)_{2}\right)_{3}\right)_{5}, \\
\sigma^{\prime}(1, m-1)=\left(4+(m+1)_{2}+\left(-(m+1)_{2}\right)_{3}+\left(1-(m+1)_{2}\right)_{3}\right)_{5} .
\end{gathered}
$$

假设 $\sigma^{\prime}(0, m-1)=\sigma^{\prime}(1, m-1)$, 则 $0=2+\left(1-(m+1)_{2}\right)_{3}$, 这是不可能的, 因为该等式右端至少为 2 ; 假设 $\sigma^{\prime}(0, m-1)=\sigma^{\prime}(0, m-2)$, 则 $(m+1)_{2}+\left(-(m+1)_{2}\right)_{3}=1+\left(-(m)_{2}\right)_{3}$, 当 $m$ 为奇数时, 则可产生矛盾 $0=3$; 当 $m$ 为偶数时, 则可产生矛盾 $3=1$.

当 $u=(n-1, m-1)$ 时, 由 $\sigma$ 及 $\sigma^{\prime}$ 的定义, 顶点 $u$ 及其相邻点在 $\sigma^{\prime}$ 下的颜色分别为

$$
\begin{gathered}
\sigma^{\prime}(n-1, m-1)=\left(2+(m+1)_{2}+\left(n+1-(m+1)_{2}\right)_{3}\right)_{5}, \quad \sigma^{\prime}(n-1, m-2)=\left(3+\left(n+1-(m)_{2}\right)_{3}\right)_{5}, \\
\sigma^{\prime}(n-2, m-1)=\left(4+(m+1)_{2}+\left(n-(m+1)_{2}\right)_{3}+\left(n+1-(m+1)_{2}\right)_{3}\right)_{5} .
\end{gathered}
$$

假设 $\sigma^{\prime}(n-1, m-1)=\sigma^{\prime}(n-2, m-1)$, 则 $(m+1)_{2}+\left(n+1-(m+1)_{2}\right)_{3}=1+\left(n+1-(m)_{2}\right)_{3}$, 当 $m$ 为偶数时, 则可产生矛盾 $0=1$; 当 $m$ 为奇数时, 则有 $(n+1)_{3}=1+(n)_{3}$, 即 $(n)_{3} \neq 2$, 这与条件 $(n)_{3}=2$ 矛盾.

由以上分析知, $u$ 与 $v$ 具有不同颜色, 即 $\sigma^{\prime}(u) \neq \sigma^{\prime}(v)$.

情况3 $d(u)=3$ 且 $d(v)=4$. 则 $u=(x, 0)$, 或 $u=(n-1, y)$, 或 $u=(x, m-1)$, 或 $u=(0, y)$.

当 $u=(x, 0)$ 时, 由 $\sigma$ 及 $\sigma^{\prime}$ 的定义, 顶点 $u$ 及其相邻点在 $\sigma^{\prime}$ 下的颜色分别为

$$
\sigma^{\prime}(x, 0)=\left((x)_{3}+(x+2)_{3}\right)_{5}, \quad \sigma^{\prime}(x, 1)=\left((x+1)_{3}+(x+2)_{3}\right)_{5} .
$$

假设 $\sigma^{\prime}(x, 0)=\sigma^{\prime}(x, 1)$, 则可导出矛盾 $0=1$.

当 $u=(n-1, y)$ 时, 由 $\sigma$ 及 $\sigma^{\prime}$ 的定义, 顶点 $u$ 及其相邻点在 $\sigma^{\prime}$ 下的颜色分别为

$$
\sigma^{\prime}(n-1, y)=\left(3+\left(n+1-(y)_{2}\right)_{3}\right)_{5}, \quad \sigma^{\prime}(n-2, y)=\left(\left(n-(y)_{2}\right)_{3}+\left(n+1-(y)_{2}\right)_{3}\right)_{5} .
$$

假设 $\sigma^{\prime}(n-1, y)=\sigma^{\prime}(n-2, y)$, 则 $3=\left(n-(y)_{2}\right)_{3}$, 这是不可能的, 因为等式右端的值不超过 2 .

当 $u=(x, m-1)$ 时, 由 $\sigma$ 及 $\sigma^{\prime}$ 的定义, 顶点 $u$ 及其相邻点在 $\sigma^{\prime}$ 下的颜色分别为

$$
\begin{gathered}
\sigma^{\prime}(x, m-1)=\left(4+(m+1)_{2}+\left(x+2-(m+1)_{2}\right)_{3}+\left(x-(m+1)_{2}\right)_{3}\right)_{5}, \\
\sigma^{\prime}(x, m-2)=\left(\left(x+2-(m)_{2}\right)_{3}+\left(x-(m)_{2}\right)_{3}\right)_{5} .
\end{gathered}
$$

假设 $\sigma^{\prime}(x, m-1)=\sigma^{\prime}(x, m-2)$, 当 $m$ 为偶数时, 则有 $(x+1)_{3}=(x)_{3}$, 这会产生矛盾 $1=0$; 当 $m$ 为 奇数时, 则有 $(x)_{3}=1+(x+1)_{3}$, 这是不可能的. 事实上, 设 $(n)_{3}=r, r=0,1,2$, 则有 $r=1+(r+1)_{3}$, 而此式无论 $r$ 取 0 , 或 1 , 或2均不成立.

当 $u=(0, y)$ 时, 由 $\sigma$ 及 $\sigma^{\prime}$ 的定义, 顶点 $u$ 及其相邻点在 $\sigma^{\prime}$ 下的颜色分别为

$$
\sigma^{\prime}(0, y)=\left(3+\left(-(y)_{2}\right)_{3}\right)_{5}, \quad \sigma^{\prime}(1, y)=\left(\left(-(y)_{2}\right)_{3}+\left(1-(y)_{2}\right)_{3}\right)_{5} .
$$

假设 $\sigma^{\prime}(0, y)=\sigma^{\prime}(1, y)$, 则 $3=\left(1-(y)_{2}\right)_{3}$, 这是不可能的, 因为该等式右端的值不超过 2 .

由以上分析知, $u$ 与 $v$ 具有不同颜色, 即 $\sigma^{\prime}(u) \neq \sigma^{\prime}(v)$. 
情况 $4 d(u)=d(v)=3$. 则 $u=(x, 0)$, 或 $u=(n-1, y)$, 或 $u=(x, m-1)$, 或 $u=(0, y)$. 当 $u=(x, 0)$ 时, 由 $\sigma$ 及 $\sigma^{\prime}$ 的定义, 顶点 $u$ 及其相邻点在 $\sigma^{\prime}$ 下的颜色分别为 $\sigma^{\prime}(x, 0)=\left((x)_{3}+(x+2)_{3}\right)_{5}, \quad \sigma^{\prime}(x-1,0)=\left((x+1)_{3}+(x+2)_{3}\right)_{5}, \quad \sigma^{\prime}(x+1,0)=\left((x)_{3}+(x+1)_{3}\right)_{5}$.

假设 $\sigma^{\prime}(x, 0)=\sigma^{\prime}(x-1,0)$, 则可导出矛盾 $0=1$; 假设 $\sigma^{\prime}(x, 0)=\sigma^{\prime}(x+1,0)$, 则可导出矛盾 $2=1$.

当 $u=(n-1, y)$ 时, 由 $\sigma$ 及 $\sigma^{\prime}$ 的定义, 顶点 $u$ 及其相邻点在 $\sigma^{\prime}$ 下的颜色分别为

$$
\sigma^{\prime}(n-1, y)=\left(3+\left(n+1-(y)_{2}\right)_{3}\right)_{5}, \quad \sigma^{\prime}(n-1, y-1)=\sigma^{\prime}(n-1, y+1)=\left(3+\left(n+1-(y+1)_{2}\right)_{3}\right)_{5} .
$$

假设 $\sigma^{\prime}(n-1, y)=\sigma^{\prime}(n-1, y-1)$ 或 $\sigma^{\prime}(n-1, y)=\sigma^{\prime}(n-1, y+1)$, 则可导出矛盾 $0=1$.

当 $u=(x, m-1)$ 时, 由 $\sigma$ 及 $\sigma^{\prime}$ 的定义, 顶点 $u$ 及其相邻点在 $\sigma^{\prime}$ 下的颜色分别为

$$
\begin{gathered}
\sigma^{\prime}(x, m-1)=\left(4+(m+1)_{2}+\left(x+2-(m+1)_{2}\right)_{3}+\left(x-(m+1)_{2}\right)_{3}\right)_{5}, \\
\sigma^{\prime}(x+1, m-1)=\left(4+(m+1)_{2}+\left(x-(m+1)_{2}\right)_{3}+\left(x+1-(m+1)_{2}\right)_{3}\right)_{5}, \\
\sigma^{\prime}(x-1, m-1)=\left(4+(m+1)_{2}+\left(x+1-(m+1)_{2}\right)_{3}+\left(x+2-(m+1)_{2}\right)_{3}\right)_{5} .
\end{gathered}
$$

假设 $\sigma^{\prime}(x, m-1)=\sigma^{\prime}(x+1, m-1)$, 则可导出矛盾 $2=1$; 假设 $\sigma^{\prime}(x, m-1)=\sigma^{\prime}(x-1, m-1)$, 则可导出 矛盾 $0=1$.

当 $u=(0, y)$ 时, 由 $\sigma$ 及 $\sigma^{\prime}$ 的定义, 顶点 $u$ 及其相邻点在 $\sigma^{\prime}$ 下的颜色分别为

$$
\sigma^{\prime}(0, y)=\left(3+\left(-(y)_{2}\right)_{3}\right)_{5}, \quad \sigma^{\prime}(0, y-1)=\sigma^{\prime}(0, y+1)=\left(3+\left(-(y+1)_{2}\right)_{3}\right)_{5} .
$$

假设 $\sigma^{\prime}(0, y)=\sigma^{\prime}(0, y-1)$ 或 $\sigma^{\prime}(0, y)=\sigma^{\prime}(0, y+1)$, 则可导出矛盾 $0=1$.

由以上分析知, $u$ 与 $v$ 具有不同的颜色，即 $\sigma^{\prime}(u) \neq \sigma^{\prime}(v)$.

综上所述, $\sigma^{\prime}$ 是 $G(n, m)$ 的正常点染色. 因此, $\sigma$ 是 $G(n, m)$ 的孪生边染色.

\section{4. 结束语}

因 $G(n, m)$ 中存在相邻的最大度点, 所以 $\chi_{t}^{\prime}(G(n, m)) \geq \chi_{a}^{\prime}(G(n, m)) \geq \Delta(G(n, m))+1$. 根据定理 2.1 与定理 2.2 的结果, 可得到以下结论: 若整数 $n, m \geq 3$ 满足 $(n)_{3}=2$ 或 $(m)_{3}=2$, 则

$$
\chi_{t}^{\prime}(G(n, m))=\chi_{a}^{\prime}(G(n, m))=\Delta(G(n, m))+1 .
$$

\section{致谢}

本文为国家民委科研项目(14XBZ018), 西北民族大学科研创新团队（图论与智能计算） 计划资助的阶段性成果之一.

\section{References}

[1] A. Eric, L. Helenius, J. Daniel and V. Jonathon, On twin edge colorings of graphs, Discussiones Mathematicae Graph Theory, vol. 34, pp. 613-627, 2014.

[2] J. L. Baril, H. Kheddouci and O. Togni, Adjacent vertex distinguishing edge-colorings of meshes and hypercubes, Australasian Journal of Combinatorics, vol. 35, pp. 89-102, 2006.

[3] Z. Zhang, L. Liu and J. Wang, Adjacent strong edge coloring of graphs, Applied Mathematics Letters, vol. 15, pp. 623-626, 2002.

[4] P. N. Balister, E. Gyo"ri, J. Lehel, and R. H. Schelp, Adjacent Vertex Distinguishing Edge-Colorings, Siam Journal on Discrete Mathematics, vol. 21, pp. 237-250, 2007.

[5] Y. Dai and Y. Bu, An Upper On The Adjacent Vertex-Distinguishing Chromatic Number of Graph, Mathematics in Economics, vol .26, pp. 107-110, 2009. 
[6] L. Zhang, W. Wang and K. W. Lih, An improved upper bound on the adjacent vertex distinguishing chromatic index of a graph, Discrete Applied Mathematics, vol.162, pp. 348-354, 2012.

[7] Y. Bu, K. W. Lih and W. Wang, Adjacent vertex distinguishing edge-colorings of planar graphs with girth at least six, Discussiones Mathematicae Graph Theory, vol. 31, pp. 429-439, 2011.

[8] C. C. Yan, D. J. Huang and W. F. Wang, Adjacent Vertex Distinguishing Edge-colorings of Planar Graphs with Girth at Least Four, Journal of Mathematical Study, vol. 45, pp. 331-341, 2012.

[9] M. Axenovich, J. Harant and J. Przybyło, et al, A note on adjacent vertex distinguishing colorings of graphs, Discrete Applied Mathematics, vol. 205, pp. 1-7, 2016.

[10]S. L. Tian, P. Chen, Y. B. Shao and Wang Q, Adjacent vertex distinguishing edge-colorings and total-colorings of the Cartesian product of graphs, Numberical Algebra, Control and Optimization, vol. 4, pp. 49-58, 2014.

[11]W. Wang and Y. Wang, Adjacent vertex distinguishing edge-colorings of graphs with smaller maximum average degree, Journal of Combinatorial Optimization, vol. 19, pp. 471-485, 2010.

[12]M. Chen and X. Guo, Adjacent vertex-distinguishing edge and total chromatic numbers of hypercubes, Information Processing Letters, vol. 109, pp. 599-602, 2009.

[13]H. Hocquard and M. Montassier, Adjacent vertex-distinguishing edge coloring of graphs with maximum degree $\Delta$, Journal of Combinatorial Optimization, vol. 26, pp. 152-160, 2013.

[14]W. Wang and Y. Wang, Adjacent vertex-distinguishing edge colorings of K4-minor free graphs, Applied Mathematics Letters, vol. 24, pp. 2034-2037, 2011.

[15]R. Diestel, Graph theory, Mathematical Gazette, vol. 173, pp. 67-128, 2000.

[16]J. A. Bondy and U. S. R. Murty. Graph Theory with Applications, New York, American Elsevier, 1976. 